\title{
Multihost Tuberculosis: Insights from the Portuguese Control Program
}

\author{
Mónica V. Cunha, ${ }^{1}$ Madalena Monteiro, ${ }^{2}$ Paulo Carvalho, ${ }^{2}$ Paula Mendonça, ${ }^{2}$ \\ Teresa Albuquerque, ${ }^{1}$ and Ana Botelho ${ }^{1}$ \\ ${ }^{1}$ INRB, I.P./LNIV-Instituto Nacional dos Recursos Biológicos/Laboratório Nacional de Investigação Veterinária, \\ Unidade de Sanidade Animal, Laboratório de Bacteriologia, Estrada de Benfica 701, 1549-011 Lisboa, Portugal \\ ${ }^{2}$ INRB, I.P./LNIV-Instituto Nacional dos Recursos Biológicos/Laboratório Nacional de Investigação Veterinária, \\ Unidade de Sanidade Animal, Laboratório de Patologia, Estrada de Benfica 701, 1549-011 Lisboa, Portugal
}

Correspondence should be addressed to Mónica V. Cunha, monica.cunha@lniv.min-agricultura.pt

Received 18 January 2011; Revised 26 March 2011; Accepted 4 May 2011

Academic Editor: Michael D. Welsh

Copyright ( 2011 Mónica V. Cunha et al. This is an open access article distributed under the Creative Commons Attribution License, which permits unrestricted use, distribution, and reproduction in any medium, provided the original work is properly cited.

This paper describes the current situation of animal tuberculosis in Portugal, reviewing the accomplishments and constraints of the 2001-2009 period. Notwithstanding the substantial progress achieved with the implementation of a comprehensive test and cull scheme, notification, postmortem inspection and surveillance at slaughterhouses, herd and animal prevalence have unexpectedly increased in 2009. In parallel, the recent awareness of tuberculosis in local free-ranging wildlife species causes concern regarding the final steps towards eradication, demanding new approaches to the existing disease control policies.

\section{The National Policy on Bovine Tuberculosis}

Although herd prevalence of bovine tuberculosis (bTB) in Portugal (0.20 in 2010) remains markedly below the values of other European countries, it is presently the fourth cause for rejection of cattle meat [1]. Apart from being an economically detrimental disease affecting the productivity and international trade of livestock [2], tuberculosis (TB) may potentially have a severe impact on protected and/or endangered wild animal species and, as a zoonosis, on human public health [3].

Considerable progress in bTB control has been observed in Portugal after the implementation of a comprehensive eradication scheme in 1992 approved by the European Union (Council Decision 92/299/CE) based on the single intradermal comparative tuberculin test (SICTT) of cattle aged over 6 weeks and the slaughter of reactor animals. The interferon- $\gamma$ test $\left(\right.$ Bovigam $\left.^{\circledR}\right)$ is used complementarily to SICTT in nonofficially TB free herds that hold animals with doubtful reactions to the intradermal test or in herds that consecutively hold positive SICTT animals (chronic bTB herd infection).
In 2001, the program became cofinanced by the European Union (in the framework of Council Directive 64/432, as amended). The National Veterinary Authority (DGV), the National Reference Laboratory (NRL-INRBLNIV), the Regional Veterinary Services Directorates, the regional laboratories from the Ministry of Agriculture, the municipal and private veterinarians from breeders organisations are the entities engaged in the bTB control program acting at the central, regional, or local level. The national program covers the whole territory of mainland Portugal, while a specific surveillance program is applied to the insular region of Azores, an important dairy region that has remained relatively untouched by the disease.

\section{The Bovine Population in Portugal}

While in the past decade, the number of existing farms in Portugal has decreased to almost half, the number of animals decreased only slightly, indicating that, on average, farms are increasing in size. In 2009, the total number of herds was 37,584 (Figure 1(a)) and the number of cattle was 1,060,831, 




(a)
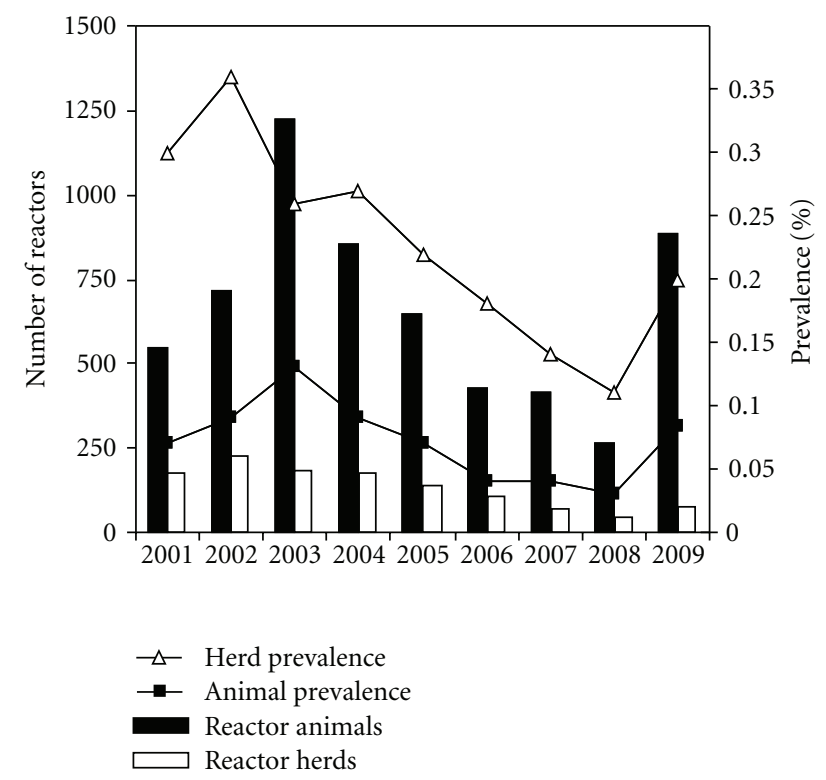

(b)

FIGURE 1: (a) Number of herds officially bTB free (white bars) and nonofficially bTB free (black bars) among the total number of herds (indicated by grey bars) in Portugal, for the period 2001-2009. Officially bTB free herds refer to herds that exclusively comprise animals exempt of disease symptoms and enclosing animals that yielded negative reactions to two consecutive official intradermal tests (applicable for cattle aged over 6 weeks). Source: [4]. (b) Number of reactor animals and reactor herds and animal/herd prevalence in Portugal for the period 2001-2009.

giving a national average of 28 animals per farm [5]. In the Northwest part of the country, the herds are usually small dairy farms, with approximately 25,000 dairy herds holding 300,000 animals. In the South-Central part, larger beef herds predominate: the Alentejo region has 4,430 beef herds comprising 523,704 animals [1].

\section{Clinical and Laboratory Evaluation of Bovine Tuberculosis}

An animal submitted to routine control testing under the National eradication plan is clinically considered bTB suspect if it yields (a) a clear SICTT-positive reaction (skin reaction of $>4 \mathrm{~mm}$ around the tuberculin injection site and/or clinical symptoms around the inoculation site such as extensive or diffuse edema, exudates, necrosis, or a pronounced inflammatory reaction of the regional lymph nodes), (b) two consecutive doubtful reactions to the intradermal test, which must be performed with an interval of, at least, 42 days, (c) a positive reaction to the interferon- $\gamma$ test, and (d) a doubtful reaction to SICTT, while belonging to an herd previously confirmed as bTB infected and holding, at least, another animal with a positive SICTT reaction in the same screening test. Animals in such conditions undergo sanitary slaughter followed by official veterinary inspection and laboratory investigation of the organs and lymphatic nodes at the NRL. Finally, only the isolation of Mycobacterium bovis or M. tuberculosis during culture for mycobacteria or the observation of typical TB lesions during histopathological analysis of the organs confirms the animal as being TB infected. Animals with postmortem suspect lesions detected at abattoir during routine slaughter are also considered $\mathrm{TB}$ suspects and undergo laboratory investigation for isolation of the agent. If the suspect animal belongs to a previously confirmed TB-infected herd, it is immediately considered infected without the need of laboratory investigation.

\section{Bovine Tuberculosis Surveillance Results: The Situation from 2001-2009}

In Portugal, since the test and slaughter policy was put in force, $99.31 \%$ herds have been officially declared free of bTB infection (Figure 1(a)) (results from 2008), meaning that all herd animals are exempt of disease symptoms, all animals aged over 6 weeks yielded a negative reaction to two consecutive official intradermal tests, and, also, all animals introduced in the herd must arise from other officially free bTB herds. The trend in herd prevalence has been slowly improving for the past decade (Figure 1(b)) in parallel with the incidence of infected animals that has been steadily decreasing, with the lowest value verified in 2008 (0.16; 264 animals) (Figure 1(b)) [4]. However, a marked heterogeneity is observed in the prevalence rates across the six affected regions of Portugal mainland: during the 2001-2009 period, the percentage of infected herds varied from $0.05-0.1$ in Beira Litoral to $0.29-1.24$ in Alentejo region [4]. These geographical differences may be related to differences in herd sizes, management practices (i.e., extensive regimen) and the presence of tuberculosis-infected wildlife favouring interspecies transmission.

Unexpectedly, in 2009, a significant increase in animal prevalence was registered: 885 infected animals were detected, corresponding to 76 infected herds (59 newly infected) (Figure 1(b)). Herd prevalence and herd incidence increased twofold, while animal prevalence increased more than threefold by comparison to 2008 [1, 4, 5] (Figure 1). Moreover, persistent breakdowns occurred consistently in some regions of mainland Portugal, where the largest livestock farms are located $[1,4]$. 
Evaluation of the reasons behind the recent increase of bTB in Portugal is not straightforward and may be a combination of genuine disease increase with higher vigilance efficacy. In fact, the increasing effort of the official authorities to raise the awareness of the various stakeholders engaged in the National bTB control program, and, consequently, to improve the antemortem and postmortem diagnosis in livestock may have biased the prevalence numbers of the last year. It is well known that the scarcity of qualified veterinary inspectors and trained personnel limits the efficiency of antemortem and postmortem examination. The effort of the authorities to train specialized personnel may have, apparently, led to an increase of the sensitivity of veterinary inspection with a greater number of specimens with visible lesions suggestive of TB being submitted to bacteriological and histopathological analysis: in 2009, 58 animals exhibiting confirmed bTB lesions were detected at abattoir, while, in the previous year, only 20 confirmed carcasses were reported [5]. In addition, during 2009, the supplier of the tuberculin used in the intradermal test was changed. Differences in the exact composition and potency of tuberculin used in the test may also have accounted for an increase of positive cases [1].

In 2009, among the 856,696 animals of the mainland submitted to the intradermal test, 716 were skin reactors, while 245 (including confirmation tests) gave positive results in the interferon- $\gamma$ test (out of 2657 tested animals) [5]. Altogether, 885 animals were reported by the National Veterinary Authority as being infected with bTB $[4,5]$. In 2009, only 22 animals that underwent sanitary slaughter presented typical tuberculous lesions during veterinary inspection; conversely, 58 animals that tested negative by the intradermal test presented suspect lesions at routine slaughter that were later confirmed as bTB [5], raising concern about the sensitivity of the test. Presently, all skin test positive animals are subjected to abattoir inspection and selected organs undergo histopathology and mycobacterial culture analysis. In 2009, 64.3 per cent of the samples sent to the NRL were confirmed as TB-positive through the isolation of M. bovis or M. caprae $[5]$.

\section{Wildlife Research}

The difficulties existing in the final eradication steps of bTB in Portugal in livestock have raised attention to TB infection in free-ranging wildlife. Depending on the region of the world, numerous species of mammals are susceptible to M. bovis and even to $M$. caprae infection [3, 6]. Potential obstacles to bTB control in livestock are the existence of reservoirs in wildlife and the putative presence of the causative agent(s) in the environment [6]. Although there is no accurate census of the population of deer and wild boar in Portugal, it is recognized that the abundance and density of these species in the territory were largely increased in the last decades [7], in parallel with the proliferation of large game hunting areas/reserves and an increase of artificial management such as fencing, feeding, and watering. A few national studies have identified two regional foci where wildlife species are infected with $M$. bovis with growing evidence of being disease reservoirs and implicated in a number of tuberculosis breakdowns in cattle [8-11]. Specifically, in Idanha-a-Nova county located in the Central East part of the country, around Tejo International River, and in Moura-Barrancos situated in the South East part of Alentejo region along Guadiana River, wild boar (Sus scrofa) and deer (mainly red deer-Cervus elaphus) are infected with $M$. bovis or $M$. caprae [8-12]. Although these two foci are geographically separated, they share common characteristics: (a) they are two of the most important hunting regions holding important densities of these two wild ungulate species that are also key game species; (b) the highest prevalence of TB in cattle is in these two regions; (c) larger beef herds and extensive management production systems predominate, favoring transmission between livestock and wildlife.

\section{Bacteriology-Based Diagnosis from 2002 to October 2010}

Organ or lymph node samples from either domestic or wild species sent to the NRL for the confirmation of TB infection undergo processing according to OIE standard procedures [13]. After homogenisation, decontamination and neutralisation, samples are inoculated onto egg-based solid and liquid media (Lowenstein-Jensen with pyruvate, Stonebrinks and BACTEC 9000) and incubated for a minimum of 8 weeks at $37^{\circ} \mathrm{C}$. Ziehl-Nielsen stains are routinely performed and species identification of presumptive mycobacterial isolates is based on the restriction fragment length polymorphism analysis of the PCR-amplified gyrB gene after digestion with $R s a I$ and SacII to distinguish M. bovis and M. caprae from the other members of Mycobacterium tuberculosis complex [14], or using the commercial reverse hybridization assays INNOLiPA Mycobacteria (Innogenetics, Belgium) or GenoType Mycobacterium (Hain diagnostics, Germany).

M. bovis is, as expected, the main species/ecotype of the Mycobacterium tuberculosis complex (MTC) isolated from the majority of animal tuberculosis cases in the country, both in domesticated cattle and in wild species. Nevertheless, $M$. caprae has also been isolated from cattle under extensive management and wild boar [12, 15], despite being primarily associated with disseminated caprine TB in goats [12]. More than thirty eight per cent of 5477 samples from suspect cattle submitted to culture during the 2002-2010 period were confirmed as TB positive. Around 1\% $(n=23)$ of the mycobacterial isolates obtained from cattle were identified as $M$. caprae while $92 \%$ were $M$. bovis. The remaining isolates belonged to Mycobacterium avium complex or to environmental nontuberculous mycobacteria. Forty per cent of the samples analyzed from farmed goat (Capra aegagrus hircus, $n=124$ ) were confirmed as caprine TB, $85 \%$ of the cases being related to $M$. caprae infection.

During the same period, 343 samples from wild boar and 544 samples from red deer were received at the NRL for the confirmation of tuberculosis. These samples invariably originated from nonfenced, free-ranging populations of deer and boar hunted in two out of most important big game hunting counties of the country: Idanha-a-Nova and Moura-Barrancos. Although veterinary inspection is not 
compulsory in hunting game, suspect samples were sent to the NRL following gross pathological evaluation performed systematically in the field by local veterinarians in hunting game activities that request official veterinarian assistance. Isolation of M. bovis or M. caprae occurred in, approximately, $51 \%$ of the tissue samples examined from red deer and $63 \%$ from wild boar. As in the case of tuberculosis in cattle, TB infection caused by $M$. bovis was definitely most frequent, although a few $M$. caprae isolates $(n=3,1.4 \%)$ were also detected in boar $[8,12]$.

\section{Insights from Molecular Epidemiology}

DNA fingerprinting has been applied to a proportion of the isolates obtained from different host species to improve our understanding of the epidemiology of the disease at the local and national level. The molecular analysis of $M$. bovis and $M$. caprae isolates obtained in Portugal from skin test reactor animals, farmed domestic animals, and wild animals has been performed by the NRL. Although, initially, DNA fingerprinting was only applied to selected isolates based on geographical, host species, and herd persistent breakdowns criteria, from 2009 onwards, genotyping is being applied to all $M$. bovis and $M$. caprae isolates. The combination of spoligotyping and MIRU-VNTR using a hierarchical two step approach has contributed to the disclosure of the prevalent $M$. bovis and $M$. caprae strains involved in TB infection [8, 10, 12, 15]. Fifty spoligotyping patterns (www.mbovis.org) have been obtained from five animal species (farmed cattle, goat and sheep, wild boar, and red deer) that resulted from the characterization of $546 \mathrm{M}$. bovis (49 patterns) and $22 \mathrm{M}$. caprae isolates (one pattern) from 2002 to 2009 (Table 1). The molecular characterization of $M$. bovis and M. caprae isolates from red deer and boar has confirmed the apparent high genotypic diversity observed in cattle isolates with the disclosure of 27 spoligotypes, some being shared with cattle, while others appear to be specific to wild hosts [8]. These molecular epidemiology studies have positively correlated the presence of certain $M$. bovis genotypes in infected red deer with bTB occurrence in cattle from the same region by the same strain (as revealed by spoligotyping and MIRU-VNTR) but also in wild boar populations, providing evidence of disease interaction between several host species [8].

The most frequent spoligotyping profiles isolated in Portugal during the 2002-2009 period was SB0121 (23\%), followed by SB0119 (12\%). The relative prevalence of the predominant $M$. bovis genotypes varies according to the animal host: SB0121 and SB0119 are the most prevalent in cattle, while, in deer, SB0121 and SB0122 predominate, whilst SB1264 and SB0121 are more frequent in the wild boar isolates genotyped so far. The pattern of SB0119 differs from SB0121 by the absence of one spacer (spacer 15). Several studies of the direct repeat region in closely related strains of $M$. tuberculosis, whose polymorphism is explored in spoligotyping, have concluded that the evolutionary trend of this region is primarily by loss of single DVRs or multiple contiguous DVRs [16]. Remarkably, ten other profiles isolated until the present moment in Portugal also exhibit a spacer deletion in relation to the profile of SB0121, suggesting the presence of a clonal complex [15]. Spoligotype SB0121 is also the most frequent pattern isolated in Spain [17], the second most predominant type in France [18] and among the top five in Italy [19]. This finding is consistent with the regular trading of animals among these Mediterranean countries. In contrast, SB0121 is infrequent in the British Isles (less than 1\%) [20], where SB0140 is prevalent (35.9\%) [20]. Interestingly, SB0140 strains have been isolated from all geographical regions of mainland Portugal. Regular cattle trading between Portugal and the British Isles occurred until 1996 (before the bovine spongiform encephalopathy epidemics-related embargo). The profile SB0120, commonly referred as BCG-like, corresponding to the most frequent type in France [18] and Italy [19], and representing 4\% of Spanish isolates [17], is relatively infrequent in Portugal $(3.2 \%)$, yet it has been isolated from three geographical regions.

MIRU-VNTR using a panel of eight validated loci, VNTR 3232, ETR-A, ETR-B, ETR-C, QUB11a, QUB11b, MIRU26, and MIRU4 [10], has been used complementarily to spoligotyping, being particularly useful to differentiate strains within common spoligotype groups. Genotyping of 337 isolates has already generated 127 allelic profiles [8, 15]. Polymorphisms are particularly marked among SB0121 strains, the most frequent and geographically dispersed spoligotype [10]. Furthermore, MIRU-VNTR has enabled tracking of multigenotype infected herds, reactivation cases, and, also, the assessment of intra- and interspecies transmission of M. bovis and/or M. caprae $[8,10]$.

\section{Geographical Differences}

The geographical localization of some of the M. bovis molecular types in the Iberian Peninsula possibly reflects localized spread of strain types and the attempt to eradicate the disease with a test and cull protocol. In addition, the historical trade of cattle and the migration of freeranging wildlife between Portugal and Spain, particularly of deer which are nonterritorial and migratory, have also each played a part in the commonalities observed among the Iberian countries. Although the spoligotype pattern of SB0121 and related strains are not unique to Portugal and Spain, cumulative data suggests that the Iberian strains may have evolved from a single common ancestor ([15, 17], unpublished results).

Regarding the characterization of domestic farmed species isolates from the mainland, the geographical region of Entre Douro e Minho (EDM), where nearly 50\% of the national herds are located, exhibits the highest strain diversity. In fact, for that region, the discriminatory power, $D$, evaluated using the Hunter and Gaston index $[21,22]$ and calculated with the website application http://insilico.ehu.es, is of 0.92 . Unexpectedly, we also found a high discriminatory index for spoligotyping in the regions where the incidence of tuberculosis is most important: Alentejo ( $\mathrm{AL}, D=0.88$ ), Trás-os-Montes (TM, $D=0.89$ ), and Beira Interior (BI, $D=0.81)$. In contrast, the lowest discriminatory index is obtained for Beira Litoral (BL, $D=0.8$ ), the less affected TB 
TABle 1: Patterns, animal host, and prevalence of the 21 most prevalent $M$. bovis and $M$. caprae spoligotypes isolated in mainland Portugal from 2002 to 2009 (joint results from $[8,15]$ ). The patterns are listed in decreasing order of prevalence.

\begin{tabular}{|c|c|c|c|c|c|c|c|}
\hline \multirow{2}{*}{ Spoligotypes } & \multicolumn{5}{|c|}{ Animal species } & \multirow{2}{*}{ Number of isolates } & \multirow{2}{*}{ Percentage of total } \\
\hline & Cattle & Goat & Sheep & Deer & Boar & & \\
\hline SB0121 & 116 & & & 10 & 4 & 130 & 22.9 \\
\hline SB0119 & 58 & & 1 & 6 & 4 & 69 & 12.1 \\
\hline SB0140 & 29 & & & 2 & & 31 & 5.5 \\
\hline SB0886 & 28 & & & & & 28 & 4.9 \\
\hline SB1090 & 21 & & & & 1 & 22 & 3.9 \\
\hline SB1095 & 21 & & & & 1 & 22 & 3.9 \\
\hline SB0157 & 7 & 12 & & & 3 & 22 & 3.9 \\
\hline SB0295 & 19 & 1 & & & 1 & 21 & 3.7 \\
\hline SB1174 & 16 & & & 3 & 1 & 20 & 3.5 \\
\hline SB1264 & 6 & & & 8 & 6 & 20 & 3.5 \\
\hline SB0265 & 9 & 1 & & 6 & 3 & 19 & 3.4 \\
\hline SB1172 & 16 & & & 2 & & 18 & 3.2 \\
\hline SB0120 & 12 & & & 5 & 1 & 18 & 3.2 \\
\hline SB0124 & 16 & & & & & 16 & 2.8 \\
\hline SB0122 & 2 & & & 10 & & 12 & 2.1 \\
\hline SB0134 & 8 & & & & & 8 & 1.4 \\
\hline SB1195 & & & & 4 & 4 & 8 & 1.4 \\
\hline SB0856 & 4 & & & 3 & 1 & 8 & 1.4 \\
\hline SB1266 & & & & 4 & 3 & 7 & 1.2 \\
\hline SB1273 & 6 & & & 1 & & 7 & 1.2 \\
\hline SB1483 & 5 & & & 1 & & 6 & 1.1 \\
\hline other & 44 & & & 9 & 3 & 56 & 9.8 \\
\hline
\end{tabular}

region. While most patterns are distributed throughout the country, a small number of patterns are restricted to specific regions [15]. Differences in herd sizes and management practices may account for differences among geographical regions. While in the most affected regions, such as Alentejo and Beira Interior, the predominant management practices are the extensive regimen and large grazing areas, where cattle farms can overlap wildlife habitats and interspecies transmission may occur, in the low TB prevalence regions, there is a considerably higher number of herds but with fewer animals and stricter confinement regimens.

The genetic relatedness of $M$. bovis isolates from wild species from regions near the border of Portugal and Spain has also suggested that contact between wild animals ranging among contiguous regions of both countries may account for a considerable number of new bTB cases.

\section{Insights from Lesion Distribution and Host Species Differences}

Pathology evaluation and the study of lesion distribution, appearance and structure of $\mathrm{TB}$ diseased animals may contribute to the understanding of $\mathrm{TB}$ disease and the associated pathogenesis, namely, as a mean to identify the routes of infection and excretion [23].

The histopathological analysis of the organs and/or lymph nodes collected from suspect animals is, at the moment, mainly (but not exclusively) performed at the NRL.
Systematic information regarding the presence of lesions in cattle in relation to the category of slaughter is reported in Table 2 for the period 2005-2009. For the same period, we present in Table 3 the number of samples exhibiting lesions confirmed as bTB by histopathology per organ and lymph node. The numbers presented are a subset of the total results of histopathology available for mainland Portugal, reporting only to the samples analyzed at the NRL in Lisbon $(n=933$ tissue samples for 2005-2009).

Considering that for 203 animals analyzed at the Lisbon Lab, there is no information available regarding the category of slaughter, at least 297 suspect animals $(31.8 \%$ out of 933) were detected during routine slaughter from 2005 to 2009 (Table 2). Among these, 170 (57.2\%) were confirmed by histopathology as bTB positive. Notably, a significant number of positive tested animals did not show any visible lesions for the period under analysis $(n=188,43.8 \%)$ (Table 2).

Regarding the macroscopic lesions observed in bovine, in our experience, lymph nodes are the most commonly affected tissue and, especially, those of the respiratory system ( $n=32.8 \%$ out of 933 samples) and also the head $(18.3 \%)$ (Table 3$)$. A variable number of lesions were observed, and these were characterised by the presence of a yellowish caseous necrosis (Figure 2(a)) accompanied by some degree of mineralization; the size of these lesions were very heterogeneous, ranging from a few millimetres to the entire organ. At the microscopic level, all lesions 
TABLE 2: Number of bTB suspect tissue samples received for histopathological analysis from cattle $(n=933)$ per year, category of slaughter, and presence or absence of bTB lesions per year as indicated by histopathology, for the period 2005-2009.

\begin{tabular}{lccccccccccccc}
\hline Category of slaughter & \multicolumn{2}{c}{2005} & \multicolumn{2}{c}{2006} & \multicolumn{2}{c}{2007} & \multicolumn{2}{c}{2008} & \multicolumn{2}{c}{2009 Total (no lesions) } \\
& & $n$ & $\%$ & $n$ & $\%$ & $n$ & $\%$ & $n$ & $\%$ & $n$ & $\%$ & \\
\hline Routine slaughter & 54 & $\mathbf{4 3}$ & 41 & $\mathbf{3 4}$ & 18 & $\mathbf{1 2}$ & 54 & $\mathbf{3 3}$ & 130 & $\mathbf{3 5}$ & $297(127)$ \\
TB eradication program & & 45 & $\mathbf{3 6}$ & 44 & $\mathbf{3 7}$ & 78 & $\mathbf{5 2}$ & 62 & $\mathbf{3 7}$ & 204 & $\mathbf{5 5}$ & $433(188)$ \\
Information not available & & 27 & $\mathbf{2 1}$ & 35 & $\mathbf{2 9}$ & 55 & $\mathbf{3 6}$ & 50 & $\mathbf{3 0}$ & 36 & $\mathbf{1 0}$ & $203(99)$ \\
\hline \multirow{2}{*}{ Total } & & $\mathbf{1 2 6}$ & & $\mathbf{1 2 0}$ & & $\mathbf{1 5 1}$ & & $\mathbf{1 6 6}$ & & $\mathbf{3 7 0}$ & $\mathbf{9 3 3 ( 4 1 4 )}$ \\
\hline \multirow{2}{*}{ Hystopathological results } & No lesions & 68 & $\mathbf{5 4}$ & 68 & $\mathbf{5 7}$ & 56 & $\mathbf{3 7}$ & 74 & $\mathbf{4 5}$ & 148 & $\mathbf{4 0}$ & 414 \\
& TB lesions & 58 & $\mathbf{4 6}$ & 52 & $\mathbf{4 3}$ & 95 & $\mathbf{6 3}$ & 92 & $\mathbf{5 5}$ & 222 & $\mathbf{6 0}$ & 519 \\
\hline
\end{tabular}

TABLE 3: Number and percentage of bovine tissue samples (organs and lymph nodes) confirmed as bTB positive by histopathology. Results concerning the period 2005-2009 are presented per year and anatomical localization.

\begin{tabular}{|c|c|c|c|c|c|c|c|c|c|c|c|c|}
\hline \multirow{2}{*}{ Year } & & \multicolumn{2}{|c|}{2005} & \multicolumn{2}{|c|}{2006} & \multicolumn{2}{|c|}{2007} & \multicolumn{2}{|c|}{2008} & \multicolumn{2}{|c|}{2009} & \multirow{2}{*}{ Total (\%) } \\
\hline & & $n$ & $\%$ & $n$ & $\%$ & $n$ & $\%$ & $n$ & $\%$ & $n$ & $\%$ & \\
\hline \multirow{8}{*}{ Affected lymph nodes } & head & 14 & 24 & 6 & 12 & 5 & 5 & 23 & 25 & 47 & 21 & $95(\mathbf{1 8 . 3 )}$ \\
\hline & thoracic & 16 & 28 & 21 & 40 & 19 & 20 & 48 & 52 & 66 & 30 & $170(32.8)$ \\
\hline & abdominal & 2 & 3 & 1 & 2 & 2 & 2 & 1 & 1 & 2 & 1 & $8(1.5)$ \\
\hline & unknown & 21 & 36 & 13 & 25 & 62 & 65 & 13 & 14 & 80 & 36 & $189(36.4)$ \\
\hline & head and thoracic & 2 & 3 & 7 & 13 & 3 & 3 & 1 & 1 & 10 & 5 & $23(4.4)$ \\
\hline & head and abdominal & 0 & 0 & 0 & 0 & 1 & 1 & 0 & 0 & 2 & 1 & $3(0.6)$ \\
\hline & thoracic and abdominal & 0 & 0 & 2 & 4 & 1 & 1 & 1 & 1 & 10 & 5 & $14(2.7)$ \\
\hline & head, thoracic, and abdominal & 0 & 0 & 0 & 0 & 1 & 1 & 0 & $\mathbf{0}$ & 0 & $\mathbf{0}$ & $1(0.2)$ \\
\hline \multirow{2}{*}{ Other organs affected } & lungs & 10 & 17 & 9 & 17 & 35 & 37 & 23 & 25 & 72 & 32 & 149 \\
\hline & liver & 0 & 0 & 2 & 4 & 2 & 2 & 6 & 6 & 11 & 5 & 21 \\
\hline
\end{tabular}

observed were characteristic of bovine tuberculosis [24] (Figure 2(b)). Granulomas observed in the lungs were, sometimes, adjacent to the bronchioles, revealing erosion of the wall and allowing leakage of the infected material into the lumen (Figure 2(c)). Ziehl-Neelsen staining of the affected tissue sections indicated a very small number of acidalcohol fast bacilli. In some of the positive tuberculin tested animals in whom no macroscopic lesions were detected, small granulomas with no necrotic core and formed only by epithelioid and multinucleated giant cells were detected (Figure 2(d)). These represent an early stage of the infection.

In red deer, the lungs and mesenteric lymph nodes were the most affected organs (Table 4) as previously described by other authors [25]. The lesions found presented a marked heterogeneity regarding their size, ranging from very small necrotic foci to large thin-walled abscesses, sometimes reaching $5 \mathrm{~cm}$ wide. In the centre of these, a pale yellowish, necrotic, and purulent material was seen (Figure 3(a)). The centre of these lesions were filled by necrotic debris, scarcely mineralized containing debris, and neutrophils which also showed signs of necrosis. A narrow layer of mononucleated macrophages, lymphocytes, and a variable number of multinucleated giant cells enveloped this core (Figure 3(b)). These lesions are usually enclosed by a fibrous capsule. The pulmonary granulomas observed were variable in terms of dimensions as well as in terms of limits definition. Some of them were confluent, and they all showed central necrosis involved by mononucleated macrophages, neutrophils, lymphocytes, and multinucleated giant cells. Sometimes, the lesions were diffuse presenting an infiltration of the lung alveoli by mononucleated macrophages, neutrophils and multinucleated giant cells (Figure 3(c)).

In wild boar, the macroscopic lesions observed indicate that the most affected organs are lymph nodes although we have no information regarding the anatomical localization of a considerable part of the analyzed samples, since they arrived to the NRL without such identification (Table 4). Well-circumscribed granulomas with central necrosis and marked mineralization were observed. Microscopically, these granulomas were characterised by the presence of a partially mineralized necrotic core involved by mononucleated macrophages, lymphocytes, and rare multinucleated giant cells and surrounded by a well-defined fibrotic capsule (Figure 3(d)).

\section{Challenges for the Future}

The surveillance reports of the last year concerning bovine tuberculosis in Portugal suggest the possibility of a (re)emergent disease, demanding the creation of a large strategic framework combining prioritized research, improved management strategies, and the consistent engagement of stakeholders. There has been a growing effort in raising awareness of the stakeholders to the importance of bTB control and to the involvement in the programmed actions of the eradication plan. This has been accomplished 


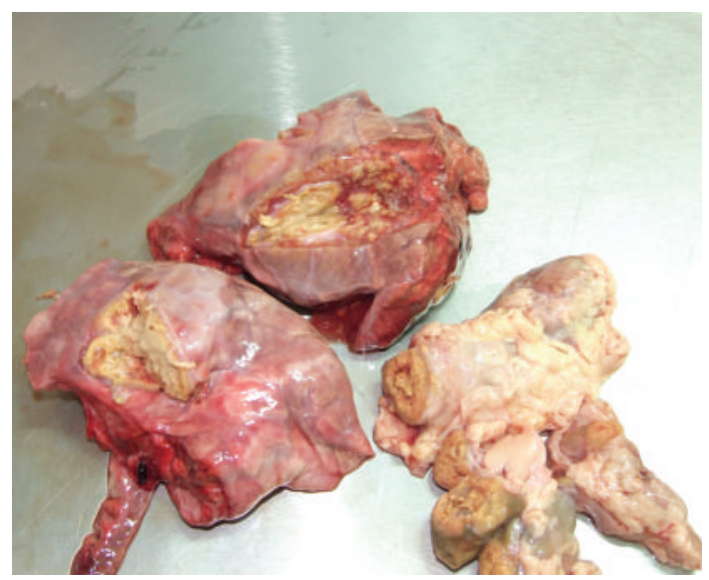

(a)

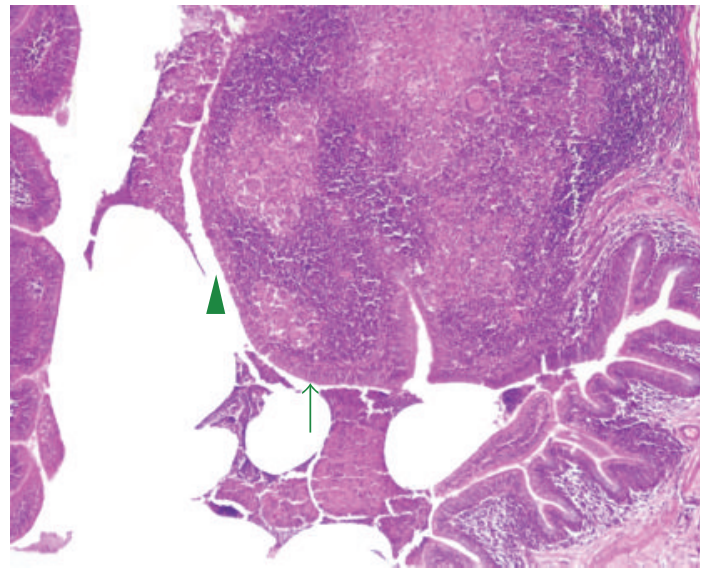

(c)

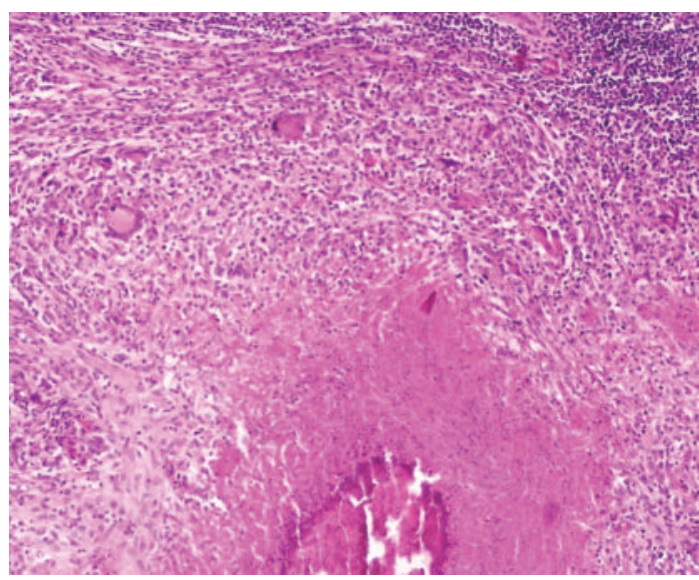

(b)



(d)

FIgURE 2: Typical macroscopic and microscopic lesions from bovine. Legend: (a) Lung and lymph node with extensive yellowish caseous necrosis; (b) Lymph node (100x) — a typical tuberculous granuloma with central necrosis, mineralization and multinucleated giant cells; (c) Lung (40x) - intrabronchial granuloma. Note the multinucleated giant cells. Arrow: bronchiolar epithelium; arrowhead: eroded bronchiolar epithelium; (d) Lymph node (100x)—microgranulomas with multinucleated giant cells and epithelioid cells.

TABLE 4: Results of the histopathological analysis of tissue samples from wild boar $(n=153)$ and red deer $(n=356)$ per year for the period 2005-2009, indicating the number of samples presenting lesions in relation to their anatomical localization.

\begin{tabular}{|c|c|c|c|c|c|c|c|c|c|c|c|c|}
\hline \multirow{2}{*}{ Year } & \multicolumn{2}{|c|}{2005} & \multicolumn{2}{|c|}{2006} & \multicolumn{2}{|c|}{2007} & \multicolumn{2}{|c|}{2008} & \multicolumn{2}{|c|}{2009} & \multicolumn{2}{|c|}{ Total } \\
\hline & Boar & Deer & Boar & Deer & Boar & Deer & Boar & Deer & Boar & Deer & Boar & Deer \\
\hline Lesions & 1 & 18 & 12 & 21 & 3 & 13 & 46 & 54 & 57 & 81 & 119 & 187 \\
\hline Lung & 1 & 8 & 1 & 10 & 0 & 4 & 1 & 26 & 3 & 29 & 6 & 77 \\
\hline Liver & 0 & 0 & 0 & 1 & 0 & 1 & 0 & 0 & 0 & 0 & 0 & 2 \\
\hline Spleen & 0 & 0 & 1 & 0 & 0 & 0 & 0 & 0 & 1 & 0 & 2 & 0 \\
\hline Lung + LMD & 0 & 3 & 2 & 1 & 0 & 0 & 0 & 5 & 2 & 6 & 4 & 15 \\
\hline HLN & 0 & 0 & 0 & 0 & 0 & 0 & 4 & 0 & 7 & 0 & 11 & 0 \\
\hline LMD & 0 & 0 & 0 & 0 & 0 & 0 & 0 & 3 & 0 & 0 & 0 & 3 \\
\hline LMES & 0 & 2 & 1 & 5 & 0 & 1 & 1 & 3 & 1 & 15 & 3 & 26 \\
\hline $\mathrm{LMD}+\mathrm{LME}$ & 0 & 0 & 0 & 1 & 0 & 0 & 0 & 0 & 0 & 0 & 0 & 1 \\
\hline NIL & 0 & 5 & 7 & 3 & 3 & 7 & 40 & 17 & 43 & 31 & 93 & 63 \\
\hline No lesions & 0 & 2 & 1 & 4 & 11 & 27 & 9 & 132 & 13 & 4 & 34 & 169 \\
\hline Total & 1 & 20 & 13 & 25 & 14 & 40 & 55 & 186 & 70 & 85 & 153 & 356 \\
\hline
\end{tabular}

Legend: LMD: mediastinal lymph nodes; LMES: mesenteric lymph nodes; HLN: head lymph nodes; NIL: nonidentified lymph nodes; Lung + LMD: lesions detected both in the lungs and in the mediastinal lymph nodes; LMD + LMES: lesions detected both in mediastinical and mesenteric lymph nodes. 


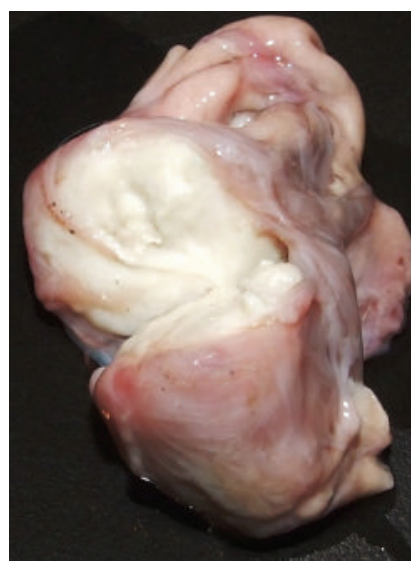

(a)

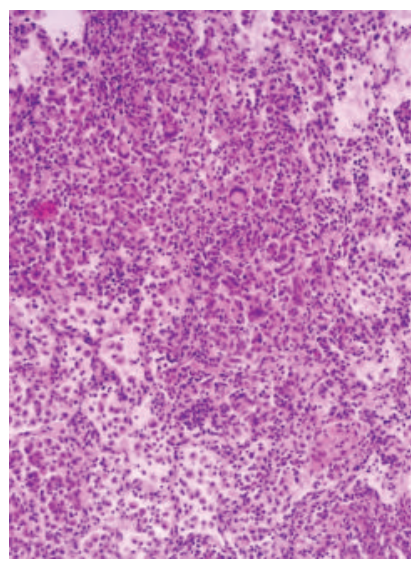

(c)



(b)

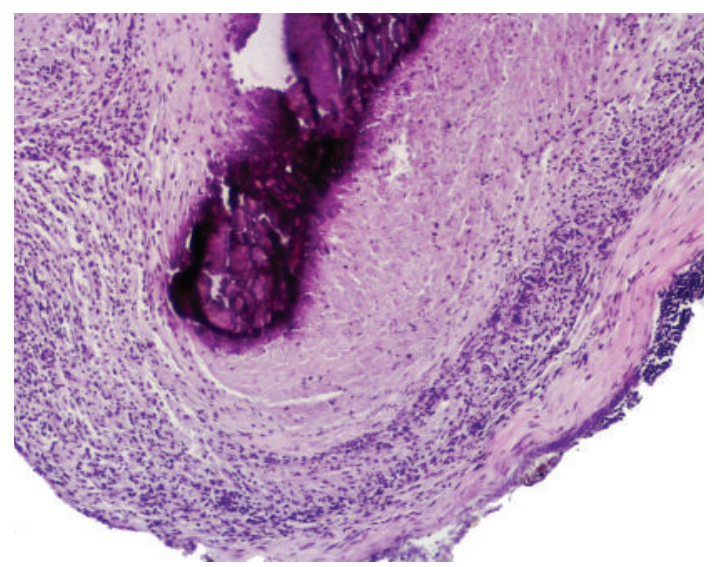

(d)

FIGURE 3: Typical lesions from deer and wild boar. Legend: macroscopic (a) and microscopic (b) images of red deer abscesses containing (a) a pale yellowish necrotic and purulent material and (b) (100x) necrotic area surrounded by epithelioid cells and multinucleated giant cells (as indicated by arrow); (c) micrograph of deer lung (100x): note the diffuse infiltration by epithelioid cells, neutrophils and multinucleated giant cells; (d) (40x) micrograph of a lymph node from wild boar: note the granuloma with extensive necrosis and mineralization.

through the education and training of veterinarians, closer followup and supervision of field veterinarians that perform the tuberculin test, training of abattoir workers and slaughterhouse inspectors, registry, control of animal movements and premovement testing, surveillance of wildlife and definition of risk areas for TB transmission, and the adoption of polyphasic laboratory practices (blood-based laboratory tests and the combination of traditional culture methods with molecular methods) to improve animal $\mathrm{TB}$ diagnosis and knowledge of the associated epidemiology. All breakdowns detected via testing or slaughterhouse inspection are investigated through epidemiological queries with the aim of determining if the infection was introduced via infected cattle. Movements are traced back. While spoligotyping was initially applied to a subset of strains as a particular initiative of the National Reference Laboratory, at the present time, in certain problematic areas, such as those that overlap wildlife, the determination of M. bovis strain type is now also requested by the National Veterinarian Authority to be compared with the local and national database.
Efforts have been undertaken to gain information on geographical distribution and prevalence rates of $\mathrm{TB}$ in certain wild species. The research findings of the last three years have contributed to define the risk areas for TB prevalence in wildlife and transmission from and to livestock, causing concern for the possibility of disease spread to other, apparently, unaffected areas. Problems in wildlife appear, so far, to be localized in the South-Central part of the country, with a greater emphasis on Tejo International region $[8,9$, 11]. Monitoring studies in wildlife hosts in other dedicated study areas should, however, be performed.

The successful eradication of TB from countries with reservoirs in wild animals requires focus on the surveillance of wildlife reservoirs of M. bovis infection. Particularly, it demands progress into the development of sensitive and specific diagnosis assays available for use in field conditions in susceptible mammal host species as an important component of the strategies to improve bTB control efforts $[3,6]$. Moreover, while bTB surveillance in cattle is homogeneous throughout the country, official veterinary inspection is not compulsory in big game-hunting activities, unless the 
meat is introduced in the commercial route. Therefore, differences in the standards of postmortem inspection by veterinarians may account for the discrepancies in the TB incidence/prevalence rates that have been observed in the wildlife surveys conducted in Portugal. Efforts should be undertaken to improve this situation. Furthermore, TB surveillance in wildlife has been almost exclusively focused on examination of samples from hunter-killed deer and boar. This method underestimates the prevalence of TB, because animals are sampled only if they present macroscopic lesions.

The acknowledgment of the presence of $\mathrm{TB}$ in wildlife in the country presents a new challenge on approaches to disease control with an impact on the existing surveillance policies. Sanitary inspection in large game species should become systematic and compulsory. A surveillance network should be established all over the country to examine deer and wild boar in other unstudied areas but also to include, in known TB problematic areas, other abundant, widely distributed, animal species, such as those that scavenge on the carcasses of potentially infected animals (e.g., foxes) and/or have important social networks (badgers) and/or live as a group. In addition, efforts to prevent contacts between wildlife and livestock in risk areas should be undertaken, especially in areas of important artificial management. Fencing should be advocated and separate feed and water for different species should be warranted to prevent disease transmission. Limiting feeding of wildlife species to reduce the overall population and aggregation should also be considered in certain, highly affected areas. Management strategies should be combined at all hierarchical levels and effective communication improved through national, regional, and local networks. Management and research outputs should become clearly available to the community. Failure to adapt control programs and management strategies to the present state of the art in livestock and wildlife may perpetrate the disease, endangering the economic investment completed so far.

\section{References}

[1] "Bovine Tuberculosis Sub-Group Task Force," Tech. Rep. SANCO/11672/2010, European Commission, Health \& Consumers Directorate-General, 2010.

[2] W. Amanfu, "The situation of tuberculosis and tuberculosis control in animals of economic interest," Tuberculosis, vol. 86, no. 3-4, pp. 330-335, 2006.

[3] A. L. Michel, B. Müller, and P. D. van Helden, "Mycobacterium bovis at the animal-human interface: a problem, or not?" Veterinary Microbiology, vol. 140, no. 3-4, pp. 371-381, 2010.

[4] Programa de Erradicação da Tuberculose Bovina de 2010, Direcção Geral de Veterinária, DGV, Portugal, 2010.

[5] Tuberculose Bovina: Relatório Técnico, Direcção Geral de Veterinária, DGV, Portugal, 2010.

[6] L. A. L. Corner, "The role of wild animal populations in the epidemiology of tuberculosis in domestic animals: how to assess the risk," Veterinary Microbiology, vol. 112, no. 2-4, pp. 303-312, 2006.

[7] J. Vingada, C. Fonseca, J. Cancela, J. Ferreira, and C. Eira, "Ungulates and their management in Portugal," in European Ungulates and Their Management in the 21st Century, M.
Apollonio, R. Andersen, and R. J. Putman, Eds., pp. 392-418, Cambridge University Press, Cambridge, England, 2010.

[8] M. V. Cunha, F. Matos, A. Canto et al., "Implications and challenges of tuberculosis in wildlife ungulates: a molecular epidemiology perspective," Research in Veterinary Science. In press.

[9] M. Vieira-Pinto, J. Alberto, J. Aranha et al., "Combined evaluation of bovine tuberculosis in wild boar (Sus scrofa) and red deer (Cervus elaphus) from Central-East Portugal," European Journal of Wildlife Research, pp. 1-13, 2011.

[10] E. Duarte, M. Domingos, A. Amado, M. V. Cunha, and A. Botelho, "MIRU-VNTR typing adds discriminatory value to groups of Mycobacterium bovis and Mycobacterium caprae strains defined by spoligotyping," Veterinary Microbiology, vol. 143, no. 2-4, pp. 299-306, 2010.

[11] N. Santos, M. Correla-Neves, S. Ghebremichael, G. Källenius, S. B. Svenson, and V. Almeida, "Epidemiology of Mycobacterium bovis infection in wild boar (Sus scrofa) from Portugal," Journal of Wildlife Diseases, vol. 45, no. 4, pp. 1048-1061, 2009.

[12] E. L. Duarte, M. Domingos, A. Amado, and A. Botelho, "Spoligotype diversity of Mycobacterium bovis and Mycobacterium caprae animal isolates," Veterinary Microbiology, vol. 130, no. 3-4, pp. 415-421, 2008.

[13] OIE, Manual of Diagnostic Tests and Vaccines for Terrestrial Animals, vol. 2, 6th edition, 2008.

[14] S. Niemann, D. Harmsen, S. Rüsch-Gerdes, and E. Richter, "Differentiation of clinical Mycobacterium tuberculosis complex isolates by gyrB DNA sequence polymorphism analysis," Journal of Clinical Microbiology, vol. 38, no. 9, pp. 3231-3234, 2000.

[15] F. Matos, M. V. Cunha, A. Canto, T. Albuquerque, A. Amado, and A. Botelho, "Snapshot of Mycobacterium bovis and Mycobacterium caprae infections in livestock in a bovine tuberculosis low incidence scenario," Journal of Clinical Microbiology, vol. 48, pp. 4337-4339, 2010.

[16] J. D. Van Embden, T. Van Gorkom, K. Kremer, R. Jansen, B. A. Van Der Zeijst, and L. M. Schouls, "Genetic variation and evolutionary origin of the direct repeat locus of Mycobacterium tuberculosis complex bacteria," Journal of Bacteriology, vol. 182, no. 9, pp. 2393-2401, 2000.

[17] S. Rodríguez, B. Romero, J. Bezos et al., "High spoligotype diversity within a Mycobacterium bovis population: clues to understanding the demography of the pathogen in Europe," Veterinary Microbiology, vol. 141, no. 1-2, pp. 89-95, 2010.

[18] N. Haddad, A. Ostyn, C. Karoui et al., "Spoligotype diversity of Mycobacterium bovis strains isolated in France from 1979 to 2000," Journal of Clinical Microbiology, vol. 39, no. 10, pp. 3623-3632, 2001.

[19] M. B. Boniotti, M. Goria, D. Loda et al., "Molecular typing of Mycobacterium bovis strains isolated in Italy from 2000 to 2006 and evaluation of variable-number tandem repeats for geographically optimized genotyping," Journal of Clinical Microbiology, vol. 47, no. 3, pp. 636-644, 2009.

[20] R. G. Hewinson, H. M. Vordermeier, N. H. Smith, and S. V. Gordon, "Recent advances in our knowledge of Mycobacterium bovis: a feeling for the organism," Veterinary Microbiology, vol. 112, no. 2-4, pp. 127-139, 2006.

[21] P. R. Hunter and M. A. Gaston, "Numerical index of the discriminatory ability of typing systems: an application of Simpson's index of diversity," Journal of Clinical Microbiology, vol. 26, no. 11, pp. 2465-2466, 1988.

[22] P. R. Hunter, "Reproducibility and indices of discriminatory power of microbial typing methods," Journal of Clinical Microbiology, vol. 28, no. 9, pp. 1903-1905, 1990. 
[23] J. P. Cassidy, "The pathogenesis and pathology of bovine tuberculosis with insights from studies of tuberculosis in humans and laboratory animal models," Veterinary Microbiology, vol. 112, no. 2-4, pp. 151-161, 2006.

[24] Jubb Kennedy and Palmer, Pathology of Domestic Animals, vol. 2, 5th edition, 2007.

[25] J. C. Rhyan and D. A. Saari, "A comparative study of the histopathologic features of bovine tuberculosis in cattle, fallow deer (Dama dama), sika deer (Cervus nippon), and red deer and elk (Cervus elaphus )," Veterinary Pathology, vol. 32, no. 3, pp. 215-220, 1995. 

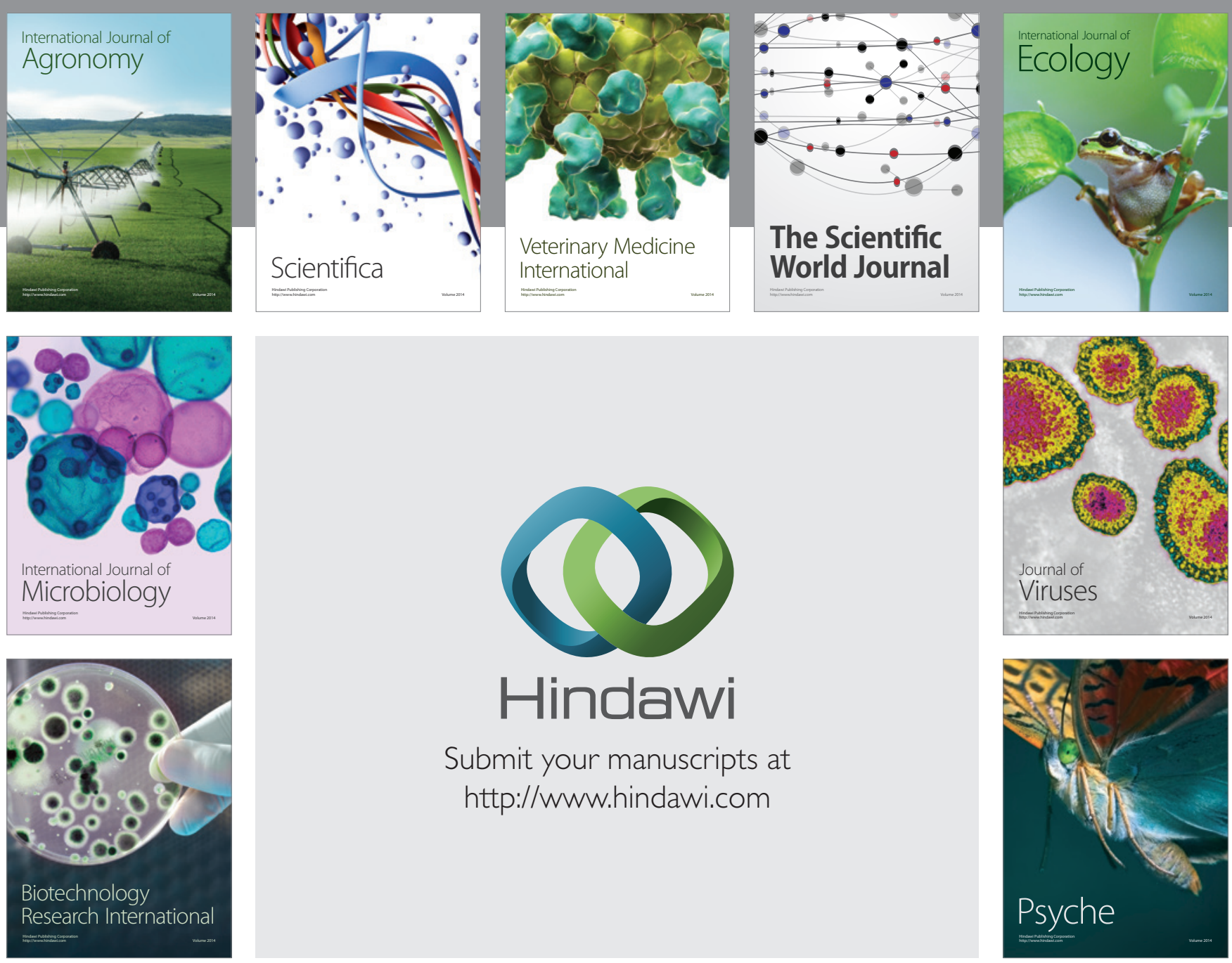

Submit your manuscripts at

http://www.hindawi.com
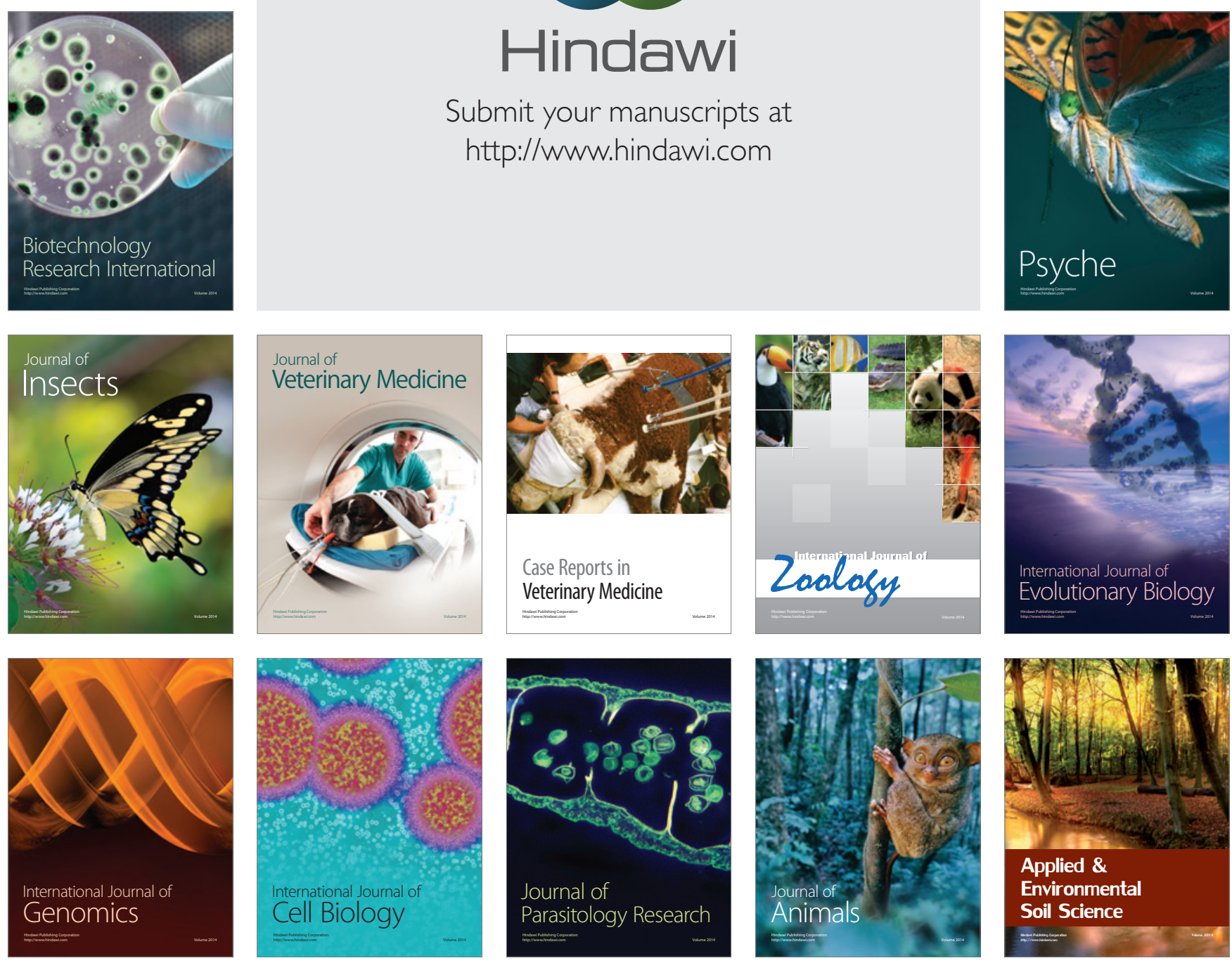